\title{
RADIUS PROBLEMS ASSOCIATED WITH PRE-SCHWARZIAN AND SCHWARZIAN DERIVATIVES
}

\author{
S. PONNUSAMY, S.K. SAHOO, AND T. SUGAWA *
}

\begin{abstract}
Some of important univalence criteria for a non-constant meromorphic function $f(z)$ on the unit disk $\mathbb{D}$ involve its pre-Schwarzian or Schwarzian derivative. We consider an appropriate norm for the pre-Schwarzian derivative, and discuss the problem of finding the largest possible $r \in(0,1)$ for which the pre-Schwarzian norm of the dilation $r^{-1} f(r z)$ is not greater than a prescribed number for normalized univalent functions $f(z)$ in the unit disk. Similar results concerning the Schwarzian derivative are also obtained.
\end{abstract}

\section{INTRODUCTION}

Let $\mathcal{A}$ (resp. $\mathcal{M}$ ) denote the set of analytic (resp. meromorphic) functions $f$ on the unit disk $\mathbb{D}=\{z \in \mathbb{C}:|z|<1\}$ normalized so that $f(0)=0$ and $f^{\prime}(0)=1$. The set $\mathcal{S}$ of univalent functions in $\mathcal{A}$ has been intensively studied by many authors. The subclass of $\mathcal{S}$ consisting of convex functions (i.e., functions mapping $\mathbb{D}$ univalently onto convex domains) is denoted by $\mathcal{K}$, and the subclass of starlike functions (i.e., functions mapping $\mathbb{D}$ univalently onto domains starlike with respect to the origin) is denoted by $\mathcal{S}^{\star}$. Thus, $\mathcal{K} \subset \mathcal{S}^{\star} \subset \mathcal{S}$. Let $\mathcal{F}$ and $\mathcal{G}$ be two subclasses of $\mathcal{A}$. If for every $f \in \mathcal{F}, r^{-1} f(r z) \in \mathcal{G}$ for $0<r \leq r_{0}$, and $r_{0}$ is the maximum value for which this holds, then we say that $r_{0}$ is the $\mathcal{G}$-radius of $\mathcal{F}$. There are many results of this type in the theory of univalent functions. See [8] for vast information in this direction. For example, the $\mathcal{K}$-radius of $\mathcal{S}$, which is usually called the radius of convexity of $\mathcal{S}$, is $r_{0}^{K}=2-\sqrt{3}=0.267 \ldots$, (cf. [7, Theorem 2.13]). On the other hand, $\mathcal{S}^{\star}$-radius in $\mathcal{S}$ (called the radius of starlikeness of $\mathcal{S})$ is $r_{0}^{\star}=\tanh (\pi / 4)=0.655 \ldots$, (cf. [7, p. 98]). Krzyż [9] applied Loewner's method to show that the radius of close-to-convexity of $\mathcal{S}$ is $r_{0}$, where the equation defining $r_{0}$ is transcendental and successive approximations yield that the lower and upper estimates for $r_{0}$ is 0.80 and 0.81 , respectively.

It is sometimes important to give univalence criteria for a non-constant meromorphic function $f$ on $\mathbb{D}$ in terms of its pre-Schwarzian or Schwarzian derivatives, that are defined by

$$
T_{f}(z)=\frac{f^{\prime \prime}(z)}{f^{\prime}(z)} \quad \text { and } \quad S_{f}(z)=T_{f}^{\prime}(z)-\frac{1}{2} T_{f}(z)^{2},
$$

respectively. Note that $T_{f}$ (resp. $S_{f}$ ) is analytic on $\mathbb{D}$ precisely when $f$ is analytic (resp. meromorphic) and locally univalent on $\mathbb{D}$. In the theory of Teichmüller spaces,

File: PoSaSu_final.tex, printed: 28-6-2018, 10.09

2010 Mathematics Subject Classification. Primary 30C45; Secondary 30C55, 33C05.

Key words and phrases. univalent functions, radius property, pre-Schwarzian and Schwarzian derivatives, pre-Schwarzian and Schwarzian norms.

* T. S. was supported in part by JSPS Grant-in-Aid for Scientific Research (B) 22340025. 
these quantities are considered as elements of complex Banach spaces as follows. For $\alpha \geq 0$, we define the norm

$$
\|\varphi\|_{\alpha}=\sup _{z \in \mathbb{D}}\left(1-|z|^{2}\right)^{\alpha}|\varphi(z)|
$$

for a meromorphic function $\varphi$ on $\mathbb{D}$. Here, we define $\|\varphi\|_{\alpha}=+\infty$ whenever $\varphi$ has a pole in $\mathbb{D}$. We denote by $\mathcal{R}_{\alpha}$ the complex Banach space consisting of analytic functions $\varphi$ on $\mathbb{D}$ with $\|\varphi\|_{\alpha}<\infty$. It is known that $f \in \mathcal{A}$ (resp. $f \in \mathcal{M}$ ) is uniformly locally univalent if and only if $T_{f} \in \mathcal{R}_{1}$ (resp. $S_{f} \in \mathcal{R}_{2}$ ). Here, a function $f$ is called uniformly locally univalent if there exists a positive constant $\rho=\rho(f)$ such that $f(z)$ is univalent on the disk $|(z-a) /(1-\bar{a} z)|<\rho$ for every $a \in \mathbb{D}$ (see, for instance, [14]).

For $\alpha \geq 0$ and $M>0$, we set

$$
\mathcal{B}_{\alpha}(M)=\left\{f \in \mathcal{A}:\left\|T_{f}\right\|_{\alpha} \leq M\right\}
$$

and

$$
\mathcal{N}_{\alpha}(M)=\left\{f \in \mathcal{M}:\left\|S_{f}\right\|_{\alpha} \leq M\right\} .
$$

As a consequence of the area theorem (see [7, p. 32]), for $f \in \mathcal{S}$ we have the inequality

$$
\left|\left(1-|z|^{2}\right) T_{f}(z)-2 \bar{z}\right| \leq 4
$$

and therefore

$$
\left|T_{f}(z)\right| \leq \frac{2(2+|z|)}{1-|z|^{2}}
$$

The last inequality leads to the implication $\mathcal{S} \subset \mathcal{B}_{1}(6)$. Note here that the Koebe function $k(z)=z /(1-z)^{2}$ satisfies the relation $\left(1-z^{2}\right) T_{k}(z)=2 z+4$, which shows the inequality (1) is sharp. On the other hand, Becker [2] showed the remarkable fact that $\mathcal{B}_{1}(1) \subset \mathcal{S}$. Sharpness of the constant 1 is due to Becker and Pommerenke [3].

For the case of Schwarzian derivative, Nehari's result [10] is fundamental: $\mathcal{N}_{2}(2) \subset$ $\mathcal{S} \subset \mathcal{N}_{2}(6)$. (The latter inclusion relation is sometimes called the Kraus-Nehari theorem.) The set $\mathcal{N}_{2}(2)$ is called the Nehari class and intensively studied by Chuaqui, Osgood and Pommerenke [5] (see also [6]). Note that the Koebe function $k(z)=z /(1-z)^{2}$ and its rotations are not contained in $\mathcal{N}_{2}(2)$ since $\left\|S_{k}\right\|_{2}=6$. It is also known that $\mathcal{K} \subset \mathcal{N}_{2}(2)$ and that the constant 2 is sharp (cf. [13, Lemma 1]).

There are variations of this type. Nehari [10] proved also that $\mathcal{N}_{0}\left(\pi^{2} / 2\right) \subset \mathcal{S}$ and Pokornyi [12] claimed that $N_{1}(4) \subset \mathcal{S}$ (see also [11]). The constants $\pi^{2} / 2$ and 4 are sharp (see [4] and [7, $\S 8.5$ or p.264], respectively). For more refinements and background, see [1].

We begin the discussion with $f \in \mathcal{S}$ and its dilations

$$
f_{r}(z)=\frac{1}{r} f(r z), \quad 0 \leq r<1 .
$$

Here, $f_{0}$ is defined as the limit of $f_{r}$; namely, $f_{0}(z)=z$. Each function $f_{r}$, together with $f$, evidently belongs to $\mathcal{S}$. Moreover, the relations

$$
T_{f_{r}}(z)=r T_{f}(r z) \text { and } S_{f_{r}}(z)=r^{2} S_{f}(r z),
$$

lead to the inequalities

$$
\left\|T_{f_{r}}\right\|_{\alpha} \leq\left\|T_{f}\right\|_{\alpha} \text { and }\left\|S_{f_{r}}\right\|_{\alpha} \leq\left\|S_{f}\right\|_{\alpha} \text { for } 0 \leq r<1 \text { and } \alpha \geq 0 .
$$


Since $T_{f_{0}}=S_{f_{0}}=0$, one expects that $\left\|T_{f_{r}}\right\|_{\alpha} \rightarrow 0$ and $\left\|S_{f_{r}}\right\|_{\alpha} \rightarrow 0$ as $r \rightarrow 0$. Thus, it may be interesting to estimate $\left\|T_{f_{r}}\right\|_{\alpha}$ and $\left\|S_{f_{r}}\right\|_{\alpha}$ and, moreover, for a given $M>0$, to find the largest possible values $r_{0}$ of $r$ such that $f_{r} \in \mathcal{B}_{\alpha}(M)$ or $f_{r} \in \mathcal{N}_{\alpha}(M)$ for all $f \in \mathcal{S}$. Note that $r_{0}$ is the $\mathcal{B}_{\alpha}(M)$-radius or the $\mathcal{N}_{\alpha}(M)$-radius of $\mathcal{S}$, respectively.

\section{Pre-Schwarzian Derivative AND RAdius PROPERTY}

We first show the following result.

Theorem 1. Let $0<r<1$ and $0<\alpha$. Set $f_{r}(z)=r^{-1} f(r z)$ for an $f \in \mathcal{S}$. Then $\left\|T_{f_{r}}\right\|_{\alpha} \leq H\left(x_{0}\right)$, where

$$
H(x)=\frac{2 r\left(1-x^{2}\right)^{\alpha}(2+r x)}{1-r^{2} x^{2}}
$$

and $x_{0}$ is the unique root of the polynomial

$$
Q(x)=r-4\left(\alpha-r^{2}\right) x-\left(1-r^{2}+2 \alpha\right) r x^{2}+4(\alpha-1) r^{2} x^{3}+(2 \alpha-1) r^{3} x^{4}
$$

in the interval $0<x<1$. Moreover, equality holds when $f$ is the Koebe function.

Proof. By (11), we have the estimate

$$
\left(1-|z|^{2}\right)^{\alpha}\left|T_{f_{r}}(z)\right|=\left(1-|z|^{2}\right)^{\alpha} r\left|T_{f}(r z)\right| \leq\left(1-|z|^{2}\right)^{\alpha} \frac{2 r(2+r|z|)}{1-r^{2}|z|^{2}}=H(|z|) .
$$

Hence, the problem reduces to finding the supremum of $H(x)$ over $0<x<1$. We now observe the formula

$$
\frac{H^{\prime}(x)}{H(x)}=\frac{Q(x)}{\left(1-x^{2}\right)(2+r x)\left(1-r^{2} x^{2}\right)} .
$$

Since $Q(0)=r>0, Q(1)=-2 \alpha\left(1-r^{2}\right)(2+r)<0$, the intermediate value theorem guarantees existence of a root of $Q(x)$ in the interval $0<x<1$. Thus, it is enough to check uniqueness of the root in $0<x<1$. To this end, we look at

$$
Q^{\prime \prime}(x)=2 r\left[6 r^{2}(2 \alpha-1) x^{2}+12(\alpha-1) r x-\left(1-r^{2}+2 \alpha\right)\right] .
$$

When $\alpha \leq 1 / 2$, obviously $Q^{\prime \prime}(x)<0$ for $0<x<1$, which implies that $Q(x)$ is concave there. Hence, the root is unique.

We now suppose that $\alpha>1 / 2$. Note first that $Q^{\prime \prime}(0)<0$. Let $x_{1}$ be the (unique) positive root of the quadratic polynomial $Q^{\prime \prime}(x)$. When $x_{1} \geq 1, Q^{\prime \prime}(x)<0$ for $0<x<1$. Thus $Q(x)$ is concave in $0<x<1$ which is enough to see the uniqueness. When $x_{1}<1$, $Q(x)$ is concave in $0<x<x_{1}$ and $Q(x)$ is convex in $x_{1}<x<1$. If $Q\left(x_{1}\right)<0$, then $Q(x)$ has a unique root in $0<x<x_{1}$ and has no root in $x_{1} \leq x<1$. If $Q\left(x_{1}\right) \geq 0$, then $Q(x)$ has no root in $0<x<x_{1}$ and has a unique root in $x_{1} \leq x<1$. At any event, the root is unique.

Remark. When $\alpha=0, Q(x)=r\left(1-x^{2}\right)\left(1+4 r x+r^{2} x^{2}\right) \geq 0$. Thus,

$$
H(x)<H(1)=\frac{2 r(2+r)}{1-r^{2}}
$$


for $0 \leq x<1$ in this case. Consequently, we have the sharp estimate

$$
\left\|T_{f_{r}}\right\|_{0} \leq \frac{2 r(2+r)}{1-r^{2}}
$$

As an application of the last theorem, at least in principle, we could find the $\mathcal{B}_{\alpha}(M)$ radius of $\mathcal{S}$. As a simple example, we obtain the following.

Theorem 2. Let $r_{0}$ be the $\mathcal{B}_{1}(1)$-radius of $\mathcal{S}$, that is, the largest possible value so that $\left\|T_{f_{r}}\right\|_{1} \leq 1$ whenever $f \in \mathcal{S}$ and $0<r \leq r_{0}$. Then $r_{0}$ is the unique root of the equation

$$
17 r^{5}-84 r^{4}-61 r^{3}-60 r^{2}+277 r-64=0
$$

in the interval $0<r<1$ and it is approximately 0.2489802 .

Proof. It is enough to solve the system of equations

$$
H(r)=\frac{2 r\left(1-x^{2}\right)(2+r x)}{1-r^{2} x^{2}}=1,
$$

and

$$
Q_{1}(x, r):=r^{3} x^{4}-\left(3-r^{2}\right) r x^{2}-4\left(1-r^{2}\right) x+r=0
$$

in the range $0<r<1$ and $0<x<1$. Since the bound in Theorem 2 is obviously increasing in $0<r<1$, such a solution is unique in this range. Let

$$
Q_{2}(x, r)=2 r\left(1-x^{2}\right)(2+r x)-\left(1-r^{2} x^{2}\right) .
$$

Then our task is to find a common zero of $Q_{1}$ and $Q_{2}$. We divide $Q_{1}$ by $Q_{2}$ with respect to $x$ to obtain

$$
4 Q_{1}(x, r)=(-2 r x+4-r) Q_{2}(x, r)+Q_{3}(x, r)
$$

where

$$
Q_{3}(x, r)=\left(9 r^{3}-8 r^{2}+4 r\right) x^{2}+\left(2 r^{3}+16 r^{2}-2 r-16\right) x+4 r^{2}-13 r+4 .
$$

Note that the set of common zeros of $Q_{1}$ and $Q_{2}$ is the same as that of $Q_{2}$ and $Q_{3}$. We repeat this procedure to have

$\left(9 r^{2}-8 r+4\right)^{2} Q_{2}(x, r)=\left(-2 r\left(9 r^{2}-8 r-4\right) x+13 r^{3}-12 r^{2}+32 r-48\right) Q_{3}(x, r)+Q_{4}(x, r)$, where

$$
Q_{4}(x, r)=-8\left(1-r^{2}\right)\left(\left(17 r^{4}-50 r^{3}+33 r^{2}-56 r+96\right) x+34 r^{3}-55 r^{2}-78 r-22\right) .
$$

By solving $Q_{4}(x, r)=0$, we have the relation

$$
x=\frac{-34 r^{3}+55 r^{2}+78 r+22}{17 r^{4}-50 r^{3}+33 r^{2}-56 r+96} .
$$

We substitute it into $Q_{3}(x, r)$ :

$$
Q_{3}(x, r)=-\frac{3\left(9 r^{2}-8 r+4\right)^{2}\left(17 r^{5}-84 r^{4}-61 r^{2}+277 r-64\right)}{\left(17 r^{4}-50 r^{3}+33 r^{2}-56 r+96\right)^{2}} .
$$

Thus we conclude that $r_{0}$ is a root of the equation in the assertion. By the uniqueness of a solution to the system in the range $0<r<1,0<x<1$, we see that such a root is unique in the interval $0<r<1$. Thus the proof is complete. 
It is also important to observe what happens in Theorem 1 as $r \rightarrow 1$. Let

$$
P(\alpha)=\sup _{f \in \mathcal{S}}\left\|T_{f}\right\|_{\alpha}
$$

Then we have the following result.

\section{Theorem 3.}

$$
P(\alpha)= \begin{cases}+\infty & \text { if } 0<\alpha<1, \\ 6 & \text { if } \alpha=1, \\ \frac{\left(2 \alpha+\sqrt{4 \alpha^{2}-6 \alpha+3}\right)(3 \alpha-3)^{\alpha-1}}{(\alpha-1 / 2)\left(\alpha-3 / 2+\sqrt{4 \alpha^{2}-6 \alpha+3}\right)^{\alpha-1}} & \text { if } 1<\alpha .\end{cases}
$$

Proof. When $\alpha=1$, the result is well-known and for $\alpha<1$, the result is obvious. We thus assume that $\alpha>1$. Let

$$
H(x)=2\left(1-x^{2}\right)^{\alpha-1}(2+x) .
$$

Then, as in the proof of Theorem 1, $P(\alpha)$ is given as the maximum of $H(x)$ over $0<x<1$. We now have

$$
\frac{H^{\prime}(x)}{H(x)}=-\frac{(2 \alpha-1) x^{2}+4(\alpha-1) x-1}{\left(1-x^{2}\right)(2+x)}=-\frac{Q(x)}{\left(1-x^{2}\right)(2+x)} .
$$

Since $Q(0)=-1<0$ and $Q(1)=6(\alpha-1)>0$, we have the unique root $x_{0}$ of $Q(x)$ in $0<x<1$ and it is indeed given by

$$
x_{0}=\frac{\sqrt{4 \alpha^{2}-6 \alpha+3}-2(\alpha-1)}{2 \alpha-1} .
$$

Noting the relation

$$
\left(2 x_{0}+1\right)\left(2 x_{0}+\frac{6 \alpha-7}{2 \alpha-1}\right)=3
$$

we have

$$
1-x_{0}^{2}=\frac{2(\alpha-1)\left(2 x_{0}+1\right)}{2 \alpha-1}=\frac{6(\alpha-1)}{2(2 \alpha-1) x_{0}+6 \alpha-7}=\frac{6(\alpha-1)}{2 \alpha-3+2 \sqrt{4 \alpha^{2}-6 \alpha+3}} .
$$

Hence, $P(\alpha)=H\left(x_{0}\right)=2\left(1-x_{0}^{2}\right)^{\alpha-1}\left(2+x_{0}\right)$ has the form given in the assertion.

By definition, $P(\alpha)$ is non-increasing in $\alpha$. Moreover, it is easy to see that $P(\alpha) \rightarrow 4$ as $\alpha \rightarrow+\infty$. We also remark that the counterpart $\tilde{P}(\alpha)$ to the Schwarzian derivative is very simple. Indeed, $\tilde{P}(\alpha)=+\infty$ if $\alpha<2$ and $\tilde{P}(\alpha)=6$ otherwise.

\section{SchWARZIAN DERIVATIVE AND RADIUS PROPERTIES}

In this section we consider the Schwarzian derivative and its norm. Our aim is to find the best possible constant $C(\alpha, r)$ for which $\left\|S_{f_{r}}\right\|_{\alpha} \leq C(\alpha, r)$ holds for $f \in \mathcal{S}$. The result can be stated in the following form. 
Theorem 4. Let $0<r<1$ and $0<\alpha$. Set $f_{r}(z)=r^{-1} f(r z)$ for an $f \in \mathcal{S}$. Then

$$
\left\|S_{f_{r}}\right\|_{\alpha} \leq \begin{cases}6 r^{2}\left(\frac{\alpha}{2 r^{2}}\right)^{\alpha}\left(\frac{1-\alpha / 2}{1-r^{2}}\right)^{2-\alpha} & \text { if } 0<\alpha<2 r^{2} \\ 6 r^{2} & \text { if } 2 r^{2} \leq \alpha .\end{cases}
$$

Equality holds when $f$ is the Koebe function.

Proof. We recall the Kraus-Nehari theorem:

$$
\left(1-|z|^{2}\right)^{2}\left|S_{f}(z)\right| \leq 6, \quad z \in \mathbb{D},
$$

for $f \in \mathcal{S}$. It is a simple exercise to see that

$$
\left(1-|z|^{2}\right)^{\alpha}\left|S_{f_{r}}(z)\right|=\left(1-|z|^{2}\right)^{\alpha} r^{2}\left|S_{f}(r z)\right| \leq 6 r^{2} K\left(|z|^{2}\right),
$$

where

$$
K(t)=\frac{(1-t)^{\alpha}}{\left(1-r^{2} t\right)^{2}}
$$

Thus, it is sufficient to find the supremum of the function $K(t)$ over $0<t<1$. We first look at the formula

$$
\frac{K^{\prime}(t)}{K(t)}=\frac{(\alpha-2) r^{2} t+2 r^{2}-\alpha}{(1-t)\left(1-r^{2} t\right)}=-\frac{\left(\alpha-2 r^{2}\right)\left(1-r^{2} t\right)+2 r^{2}\left(1-r^{2}\right)}{(1-t)\left(1-r^{2} t\right)} .
$$

When $\alpha \geq 2 r^{2}$, obviously $K^{\prime}(t) \leq 0$ in $0<t<1$. Therefore, in this case, $K(t)$ is nonincreasing in $t$ and its supremum is $K(0)=1$. On the other hand, when $0<\alpha<2 r^{2}$, the function $K(t)$ takes its maximum at

$$
t_{0}=\frac{2 r^{2}-\alpha}{(2-\alpha) r^{2}}=\frac{2 r^{2}-\alpha}{2 r^{2}-\alpha r^{2}}
$$

Since

the conclusion follows.

$$
K\left(t_{0}\right)=\left(\frac{\alpha\left(1-r^{2}\right)}{r^{2}(2-\alpha)}\right)^{\alpha}\left(\frac{2-\alpha}{2\left(1-r^{2}\right)}\right)^{2}
$$

The above theorem determines the value of

$$
C_{\alpha}(r)=\sup _{f \in \mathcal{S}}\left\|S_{f_{r}}\right\|_{\alpha}
$$

When $\alpha=0$, the above computation tells us that $K(t)$ is increasing in $0<t<1$. Therefore, $C_{0}(r)=6 r^{2} K(1)=6 r^{2}\left(1-r^{2}\right)^{-2}$. We thus summarize the conclusions:

$$
C_{\alpha}(r)= \begin{cases}\frac{6 r^{2}}{\left(1-r^{2}\right)^{2}} & \text { if } \quad \alpha=0 \\ 6 r^{2}\left(\frac{\alpha}{2 r^{2}}\right)^{\alpha}\left(\frac{1-\alpha / 2}{1-r^{2}}\right)^{2-\alpha} & \text { if } 0<\alpha<2 r^{2} \\ 6 r^{2} & \text { if } 2 r^{2} \leq \alpha .\end{cases}
$$

In particular, we observe that $C_{\alpha}(r)$ is strictly increasing in $0<r<1$. In particular, we have the following. 
Corollary 1. Let $0<M \leq 3 \alpha$. Then the $\mathcal{N}_{\alpha}(M)$-radius of $\mathcal{S}$ is $\sqrt{M / 6}$.

Proof. Let $r_{0}=\sqrt{M / 6}$. Then $2 r_{0}^{2}=M / 3 \leq \alpha$ and thus Theorem 4 implies $C_{\alpha}\left(r_{0}\right)=$ $6 r_{0}^{2}=M$.

For instance, we easily have the following:

(1) The $\mathcal{N}_{2}(2)$-radius of $\mathcal{S}$ is $1 / \sqrt{3}=0.5773 \ldots$

(2) The $\mathcal{N}_{0}(M)$-radius of $\mathcal{S}$ is obtained by solving the equation

$$
C_{0}(r)=\frac{6 r^{2}}{\left(1-r^{2}\right)^{2}}=M
$$

which gives

$$
r=\sqrt{\frac{M+3-\sqrt{9+6 M}}{M}} .
$$

(3) The $\mathcal{N}_{0}\left(\pi^{2} / 2\right)$-radius of $\mathcal{S}$ is $\sqrt{1+\left(6 / \pi^{2}\right)-\left(2 \sqrt{9+3 \pi^{2}} / \pi^{2}\right)}=0.5905 \ldots$

(4) Finally, we find $\mathcal{N}_{1}(4)$-radius of $\mathcal{S}$. In this case, we solve the equation

$$
C_{1}(r)=\frac{3}{2\left(1-r^{2}\right)}=4
$$

to obtain $r=\sqrt{5 / 8}=0.7905 \ldots$

\section{REFERENCES}

1. F. G. Avhadiev and L. A. Aksent'ev, Fundamental results on sufficient conditions for the univalence of analytic functions (Russian), Uspehi Mat. Nauk 30(1975), no. 4 (184), 3-60, English translation in Russian Math. Surveys 30(1975), 1-64.

2. J. Becker, Löwnersche Differentialgleichung und quasikonform fortsetzbare schlichte Funktionen, J. Reine Angew. Math. 255(1972), 23-43.

3. J. Becker and Ch. Pommerenke, Schlichtheitskriterien und Jordangebiete, J. Reine Angew. Math. 354(1984), 74-94.

4. M. Chuaqui, A unified approach to univalence criteria in the unit disc, Proc. Amer. Math. Soc. 123(1995), 441-453.

5. M. Chuaqui, B. Osgood, and Ch. Pommerenke, John domains, quasidisks, and the Nehari class, J. Reine Angew. Math. 471 (1996), 77-114.

6. M. Chuaqui and Ch. Pommerenke, Characteristic properties of Nehari functions, Pacific J. Math. 188(1999), 83-94.

7. P. L. Duren, Univalent Functions, Springer-Verlag, 1983.

8. A. W. Goodman, Univalent Functions, 2 vols., Mariner Publishing Co. Inc., 1983.

9. J. G. Krzyż, The radius of close-to-convexity within the family of univalent functions, Bull. Acad. Polon. Sci. 10(1962), 201-204.

10. Z. Nehari, The Schwarzian derivative and schlicht functions, Bull. Amer. Math. Soc. 55(1949), 545551.

11. S Some criteria of univalence, Proc. Amer. Math. Soc. 5(1954), 700-704.

12. V. V. Pokornyi, On some sufficient conditions for schlichtness (Russian), Dokl. Akad. Nauk SSSR 79(1951), 743-746.

13. M. S. Robertson, Univalent functions $f(z)$ for which $z f^{\prime}(z)$ is spirallike, Michigan Math. J. 16(1969), $97-101$. 
14. T. Sugawa, The universal Teichmüller space and related topics, Proceedings of the International Workshop on Quasiconformal Mappings and their Applications (India) (T. Sugawa S. Ponnusamy and M. Vuorinen, eds.), Narosa Publishing House, 2007, pp. 261-289.

Department of Mathematics, Indian Institute of Technology Madras, Chennai - 600 036, INDIA

E-mail address: samy@iitm.ac.in

Department of Mathematics, Indian Institute of Technology Indore, M-Block, I.E.T. DAVV Campus, Khandwa Road, Indore 452 017, M.P., India

E-mail address: swadesh@iiti.ac.in

Graduate School of Information Sciences, Tohoku University, Aoba-ku, Sendai 9808579, JAPAN

E-mail address: sugawa@math.is.tohoku.ac.jp 